\title{
Editorial
}

Clin Shoulder Elbow 2022;25(1):1-2

Clinics in Shoulder and Elbow

https://doi.org/10.5397/cise.2022.00857

eISSN 2288-8721

\section{Appreciation to reviewers}

\section{Young-Kyu Kim}

Department of Orthopedic Surgery, Gil Medical Center, Gachon University College of Medicine, Incheon, Korea

Many reviewers readily shared their knowledge and contributed their passion for Clinics in Shoulder and Elbow, along with their advice and suggestions for improving the quality of the manuscripts submitted for publication. The peer reviewers listed below readily gave their effort and time.

The editorial committee would like to show appreciation to all reviewers participating in this process.

Luciano Bigliani
Kerem Bilsel
Chih-Hwa Chen
Bancha Chernchujit
Chul-Hyun Cho
Nam-Su Cho
kiyong Choi
Sungwook Choi
Yong-Min Chun
Seok Won Chung
Moustafa Ismail Ibranim Elsayed
Michael Hantes
Jung-Taek Hwang
Yoon-Suk Hyun
Suk-Hwan Jang
Yoonsang Jeon
Hyeon Jang Jeong
Jeung Yeol Jeong
Jinyoung Jeong
Woong-Kyo Jeong
Jong-Hun Ji

Chunyan Jiang
Chris H. Jo
Kyu-Hak Jung
Suk Woong Kang
Doo-Sup Kim
Du Han Kim
Eugene Kim
Hyungsuk Kim
Jae Yoon Kim
Jung han Kim
Myung-Seo Kim
Myung-Sun Kim
Sae-Hoon Kim
Seong-Hun Kim
Sucheol Kim
Sung-Jae Kim
Youngbok Kim
Sang-Hun Ko
Kyoung-Hwan Koh
Chae-Gwan Kong
Jae-Man Kwak

Correspondence to: Young-Kyu Kim

Department of Orthopedic Surgery, Gil Medical Center, Gachon University College of Medicine, 21 Namdong-daero 774beon-gil, Namdong-gu, Incheon 21565, Korea

Tel: +82-32-460-3384, Fax: +82-32-423-3384, E-mail: kykhyr@gilhospital.com, ORCID: https://orcid.org/0000-0001-5672-505X

Financial support: None.

Conflict of interest: None.

Copyright@ 2022 Korean Shoulder and Elbow Society.

This is an Open Access article distributed under the terms of the Creative Commons Attribution Non-Commercial License (http://creativecommons.org/licenses/by-nc/4.0/) which permits unrestricted non-commercial use, distribution, and reproduction in any medium, provided the original work is properly cited. 
Bong Gun Lee

Doo-Hyung Lee

Hyo-Jin Lee

Jae Hoo Lee

Sang-Uk Lee

Woo Seung Lee

Ye Hyun Lee

Yongbeom Lee

Tae Kang Lim

William Levine

Andri Maruli Tua Lubis

Edward Mcfarland

Teruhisa Mihata

Tomoyuki Mochizuki

Jun-Gyu Moon

Kyu-Cheol Noh

Young-Min Noh
Kyung Soo Oh

Hyung-Bin Park

In Park

Sung-Min Rhee

In Hyeok Rhyou

Joong-Bae Seo

Sang-Jin Shin

Dongju Shin

Min Soo Shon

Hyun-Seok Song

Sung Il Wang

W.Jaap Willems

Jae Chul Yoo

Jong Pil Yoon

Hyung Moon Yoon

Tae-Hwan Yoon 